\title{
Influence of extremely low energy radiation on artificial tissue: Effects on image quality and superficial dose
}

\author{
M.-Ali H. Al-Akhras ${ }^{\mathrm{a}, *}$, K. Aljarrah ${ }^{\mathrm{a}}$, A. Al-Omari ${ }^{\mathrm{b}}$, H.M. Al-Khateeb ${ }^{\text {a }}$, B.A. Albiss ${ }^{\mathrm{a}}$, \\ K. Azez ${ }^{\mathrm{a}}$ and G. Makhadmeh ${ }^{\mathrm{a}}$ \\ ${ }^{a}$ Bio-Medical Physics Laboratory, Department of Physics, Jordan University of Science \& Technology \\ (JUST), Irbid 22110, Jordan \\ ${ }^{\mathrm{b}}$ Department of General and Pediatric Surgery, Faculty of Medicine, Jordan University of Science \& \\ Technology (JUST), Irbid 22110, Jordan
}

\begin{abstract}
The design and slicing technique of artificial soft tissue are presented. Artificial soft tissue has optical penetration properties similar to biological tissues. The soft tissues are made of agar dissolved in water as a transparent tissue (control) incorporated with scatter materials such as polystyrene microspheres and absorbers such as artificial dairy substitute, coffee mate (Carnation Co.). The radiation's interaction with 20 and $40 \mathrm{keV}$ X-ray, and visible light (400-800 nm) with different types of tissue phantoms has been investigated. The half value layer (HVL), attenuation coefficient, energy density and penetration depth through the artificial tissues has been calculated. X-ray radiation depth show significant reduction in soft tissue incorporated with polystyrene microspheres. At extremely low energy $(E)$, the half value layer decreases with increasing the energy, while the attenuation coefficient increase. The calculated values of the half value layers are in very good agreement with experimental results. The calculated values of effective linear attenuation coefficient, are found to be $\mu_{\text {eff }}(0.22-0.42)$. Significant reduction in superficial dose with clear image is found with $10 \mathrm{~mm}$ soft tissue filter used. These results suggests: possible enhancement in diagnostic imaging and reduction in excess dose to patients; artificial soft tissue can be used as filter substitute.
\end{abstract}

Keywords: Tissue phantom, penetration depth, attenuation coefficient, half value layer, photodynamic therapy

\section{Introduction}

For the last few decades, extensive work has been done to investigate and develop photodynamic therapy and to minimize the superficial dose from diagnostic beams. It is well known that extremely low energy $(E)$ is absorbed in the first few millimeters of the tissue without useful help in imaging and diagnosing. A small amount of penetration and absorption of low energy photons incident on tissue is the main objective in optical penetration for diagnostic and photodynamic therapy. The penetration depth and the mean path length provide useful information for superficial therapy treatment and diagnostic of disorders, measurements of tissue oxygenation, and functional imaging of muscle and brain. The depth reached by penetrating photons provides information on the volume of investigated tissue, while the mean path is found to be related to the sensitivity and to local variations in the absorption [1]. The human tissue is quite transparent to shorter wavelengths. The photons interactions with tissue have various

\footnotetext{
${ }^{*}$ Corresponding author: M.-A.H. Al-Akhras, Bio-Medical Physics Laboratory, Department of Physics, Jordan University of Science \& Technology (JUST), P.O. Box 3030, Irbid 22110, Jordan. E-mail: alakmoh@ just.edu.jo.
} 
penetration depths which depend on the energy of the used source. In this work the penetration depth, interaction, attenuation and some optical properties has been calculated and discussed. The individual absorption of radiation starts as soon as the photons penetrate through the tissue. The process is progressive and continues as the radiation penetrates deeper into the material. The relationship between the penetrating intensity $\left(I_{x}\right)$ and the thickness of the tissue phantom $(x)$ can be described as:

$$
I_{x}=I_{0} \mathrm{e}^{-\mu x},
$$

where $I_{0}$ is the initial intensity of the beam and $\mu$ is the linear attenuation coefficient. The depth at which the material attenuate the incident beam by one half of the initial intensity is called the half value layer $(\mathrm{HVL}=0.693 / \mu)$ for that material. The HVL represents the penetration ability of the photons and useful information can be obtained. This study emphasized the characteristics of the radiations interaction with matter (tissues) with additives like absorber and scatterer materials to serve as a key and initial step for a wide range of irradiation therapy. Also, one of the major goals in this study is to improve the image quality and to reduce the entrance dose. Evaluating the effect of a blood base tissue-like filter on the image quality of a phantom is addressed as well. A test of a phantom hand images were digitized for validation.

\subsection{Visible light}

Light interacts with tissue through different mechanisms which depend on the light wavelength and tissue structure. Mainly two types of interactions could determine the light propagation into the tissue; scattering interaction from the inhomogeneities of a cellular structure such as polystyrene microspheres in the sample and absorption interaction in the absorbing constituents such as artificial dairy substitute, coffee mate (Carnation Co.). The intensity of the scattered light depends upon the size and shape of the inhomogeneities relative to the wavelength of light. For phantoms consist of small particles with dimensions less than, roughly, one-tenth of the wavelength, scattering in general is weak. On the other hand the scattering significantly occurs when the wavelength of light is comparable to the dimensions in the structure of the tissue [2].

\subsection{X-ray}

Another source of radiation used in this work is low energy beam of X-ray $(20,30$ and $40 \mathrm{kV})$ which is considered to be one of the most useful forms of low photon energy in diagnostic imaging and therapy. $\mathrm{X}$-rays have some of the shortest wavelengths and highest energies among all kinds of electromagnetic radiation. X-rays have very high energy penetration depth in matter compare to visible light. Absorption by a substance can take place when the photons transfer their energies to the orbital electrons in the atoms and these electrons deposited their energy to the medium. The percentage energy absorbed (deposited) by the material can cause chemical, biological, and physical changes. Absorption is dependent upon the incident energy, material density and atomic number.

\section{Material and methods}

\subsection{Preparation of the samples}

Soft tissues were made with agar aqueous solutions, doped with the artificial dairy substitute, coffee mate, as a good absorber, and polystyrene microspheres. Aqueous suspensions of $5 \mathrm{~g}$ Agar diluted with 


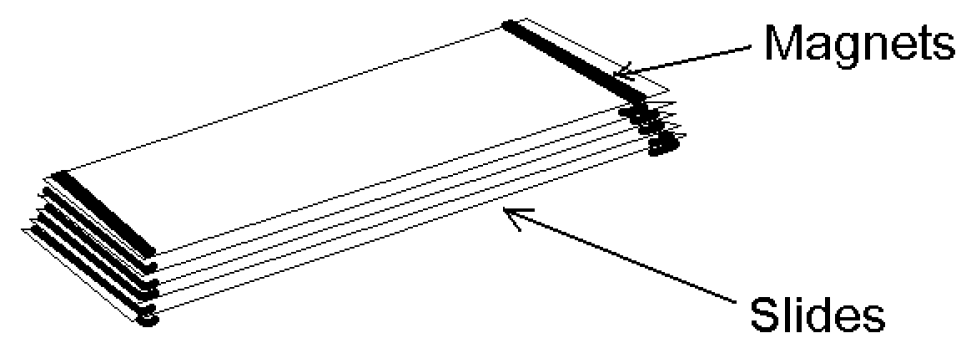

Fig. 1. Slicing and sampling technique.

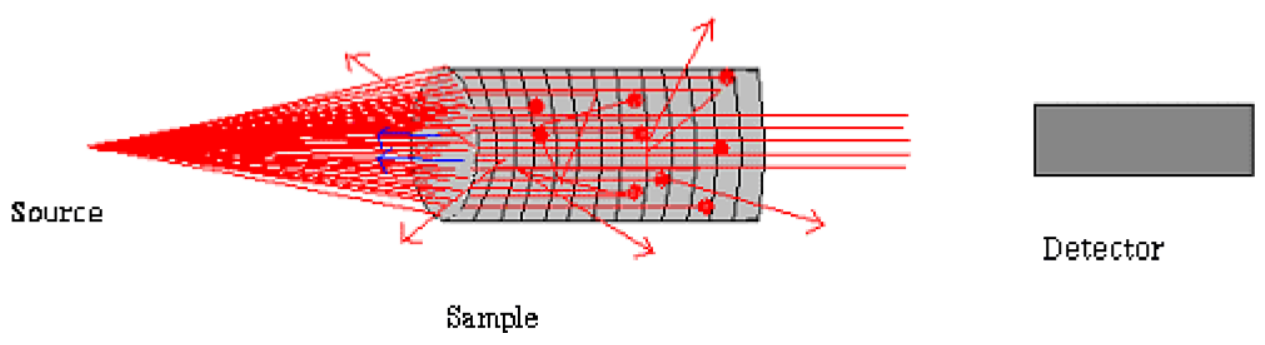

Fig. 2. Sample irradiation.

$84 \mathrm{ml}$ hot distilled water was made as control sample. The solutions were heated while stirring for about 2 min to dissolve the agar and get clear homogenous tissue and free of bubbles. To make thin layers of this mixture, microscopic slides, separated by magnet strips of $1 \mathrm{~mm}$ thickness between the two far edges, were immersed inside the heated mixture. The solutions soak up and fill all space and gaps between the slides. The magnetic strips are used to keep a $1 \mathrm{~mm}$ gap between the slides and to tight the slides together as shown in Fig. 1. The mixture together with the immersed slides was cooled to low temperature for few minutes, and then the microscopic slides were removed to obtain $1 \mathrm{~mm}$ thick slides of the soft tissue. The tissue slices were cut into a uniform circular disks and joined to gather to form a cylindrical shape as shown in Fig. 2. Similar samples were made using very low concentration of polystyrene microspheres $(0.3 \mu \mathrm{g} / \mathrm{ml})$, in order to fulfill the real scattering probability of the biological tissues. The concentration of the additives in the samples were as follows, $5.95 \mathrm{mg} / \mathrm{ml} \mathrm{Agar,} 1.19 \mathrm{mg} / \mathrm{ml}$ Coffee mate and $0.3 \mu \mathrm{g} / \mathrm{ml}$ polystyrene microspheres.

\subsection{Sample irradiation with visible light}

For intensity profile measurements, a setup is arranged as shown in Fig. 2 and a direct light is used to irradiate the sample. The used light source is the spectrophotometer PYE UNICAM PU 8600 UV/VIS at a wavelength of $550 \mathrm{~nm}$. The cuvette holder was removed and the sample layers were placed on the front window of the exiting light. The circular layers were mounted on a microscopic slide one by one. The microscopic slide was used as a background correction. The air bubbles between the layers are kicked-out and the beam was incident normally to the tip of the sample plane to minimize the amount of scattering. The transmitted beam was measured against the thickness.

\subsection{Sample irradiation with $X$-ray}

The X-ray unit apparatus (P580 TEL-X-OMETER, Teltron ltd., London, England) complete with a rate meter or counter with an integrated high-voltage supply is used. The high-voltage with $20 / 30 \mathrm{kV}$ 
DC $10 \%$ switchable electrically stabilized is used. High-vacuum X-ray tube with a copper anode, in a borosilicate-glass mantle equipped with a concave ray-outlet window with thin walls. The X-radiation focal spot of the anode is $5 \times 1 \mathrm{~mm}$. The crystal post was removed and the samples were places on the swivel arm of the counter tube in front of the detector (55901 GMBH LEYBOLD, Germany). The $\mathrm{X}$-ray source, sample and detector were aligned in a straight line as shown in Fig. 2. The beam was kept normal to the surface of the sample to prevent the beam deflection. The readings from digital counter versus thickness were recorded.

\subsection{Density measurements with artificial soft tissue}

To evaluate the possible application of this artificial soft tissue in medical imaging, a $10 \mathrm{~mm}$ blood base tissue strip of the same composition was prepared. The tissue strip was used to study the reduction of the entrance dose while achieving the same image quality. The diagnostic X-ray tube of $40 \mathrm{keV}$ in our clinic is used as a beam source. The positions of the tissue filter strip together with the Aluminum filter were interchanged and combined as shown in Table 2. The densities of the entrance beam are recoded on an X-ray film which located at $70 \mathrm{~cm}$ source to surface distance (SSD). The image quality for a phantom hand was digitized with different filter combinations and locations.

\section{Results and discussions}

\subsection{Visible light}

Compare to control, all samples show less light penetration. The spectrophotometer light shows higher penetration in the control sample (Agar) as compared with Coffee mate added to the sample.

Lower penetration in the sample of polystyrenes and polystyrenes with Coffee mate was found as shown in Fig. 3 and Table 2 (visible light source). The experimental data points of the fluence rate are exponentially decreases as depth increase. The effective linear attenuation coefficients $\left(\mu_{\text {eff }}\right)$ presented

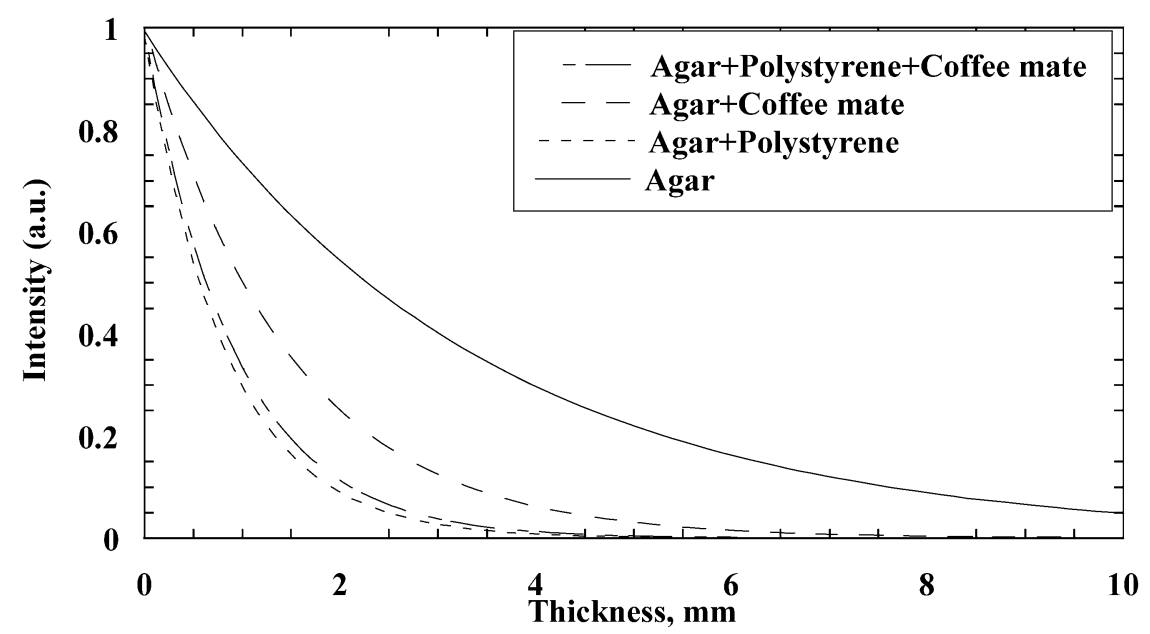

Fig. 3. Penetration of light intensity in tissue phantoms made of Agar + Coffee mate, Agar + polystyrene, Agar + Coffee mate + polystyrene and agar (as control). 
in Table 1 are obtained by the logarithmic decay of the fluence rate measured at different depths in the phantom exposed to uniform and normal beam [3].

In comparison with control (Agar) in Fig. 3, the result of these curves shows lower deep penetration for tissue to which Coffee mate has been incorporated while more lower penetration for tissue mixed with polystyrene microspheres and microspheres plus Coffee mate with no significant change in both samples contains microspheres. Therefore, multiple scattering inside the tissue takes place preventing deep penetration in case of polystyrene microspheres.

Table 1 and Fig. 4 shows the energy decreasing with increasing HVL while the linear attenuation coefficient is decreasing. HVL is the most frequently used factor for describing the penetration ability of specific radiations as well as the penetration through specific tissues. HVL is defined as the thickness of tissues penetrated by one half of radiation. Experimental values $\left(\mathrm{HVL}_{\mathrm{exp}}\right)$ are obtained from the

Table 1

Effect of extremely low energy $(E)$ of the light on optical penetration depth $\delta(\mathrm{mm})$ and HVL in soft tissue phantom

\begin{tabular}{|c|c|c|c|c|}
\hline$E\left(\times 10^{-3} \mathrm{keV}\right)$ & $\mu_{\mathrm{eff}}\left(\mathrm{mm}^{-1}\right)$ & $\delta(\mathrm{mm})$ & $\mathrm{HVL}_{\exp }(\mathrm{mm})$ & $\mathrm{HVL}_{\mathrm{th}}(\mathrm{mm})$ \\
\hline 3.10 & 0.41740 & 2.3958 & 1.6453 & 1.6603 \\
\hline 2.76 & 0.35760 & 2.7964 & 1.8566 & 1.9379 \\
\hline 2.48 & 0.26790 & 3.7327 & 2.5962 & 2.5867 \\
\hline 2.25 & 0.30290 & 3.3014 & 2.2792 & 2.2878 \\
\hline 2.07 & 0.25120 & 3.9809 & 2.4377 & 2.7164 \\
\hline 1.91 & 0.24870 & 4.0209 & 2.7018 & 2.7865 \\
\hline 1.77 & 0.22740 & 4.3975 & 2.8603 & 3.0475 \\
\hline 1.65 & 0.21760 & 4.5956 & 2.9660 & 3.1847 \\
\hline
\end{tabular}

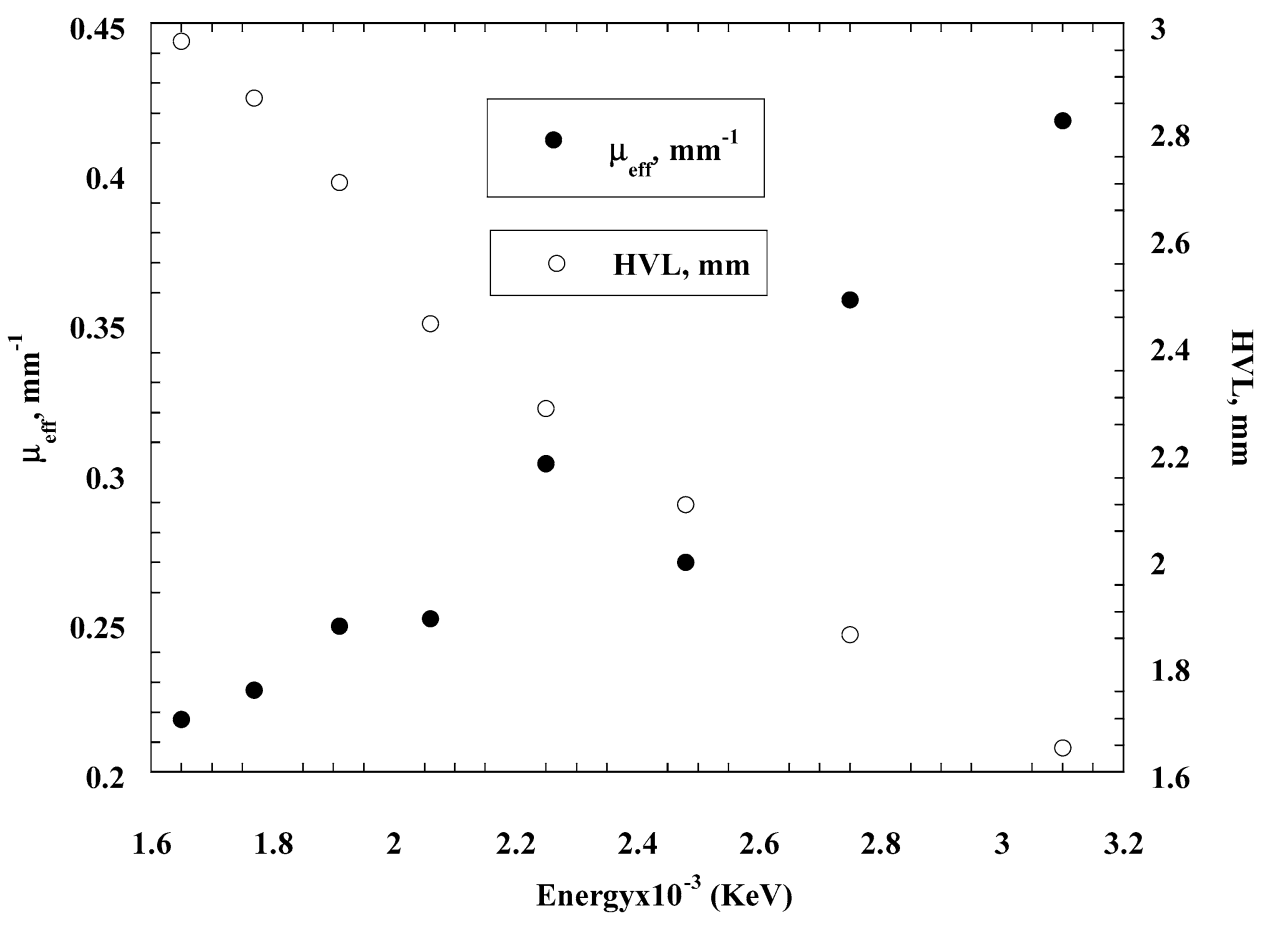

Fig. 4. Relationship between the linear attenuation coefficient and the HVL for a soft tissue. 


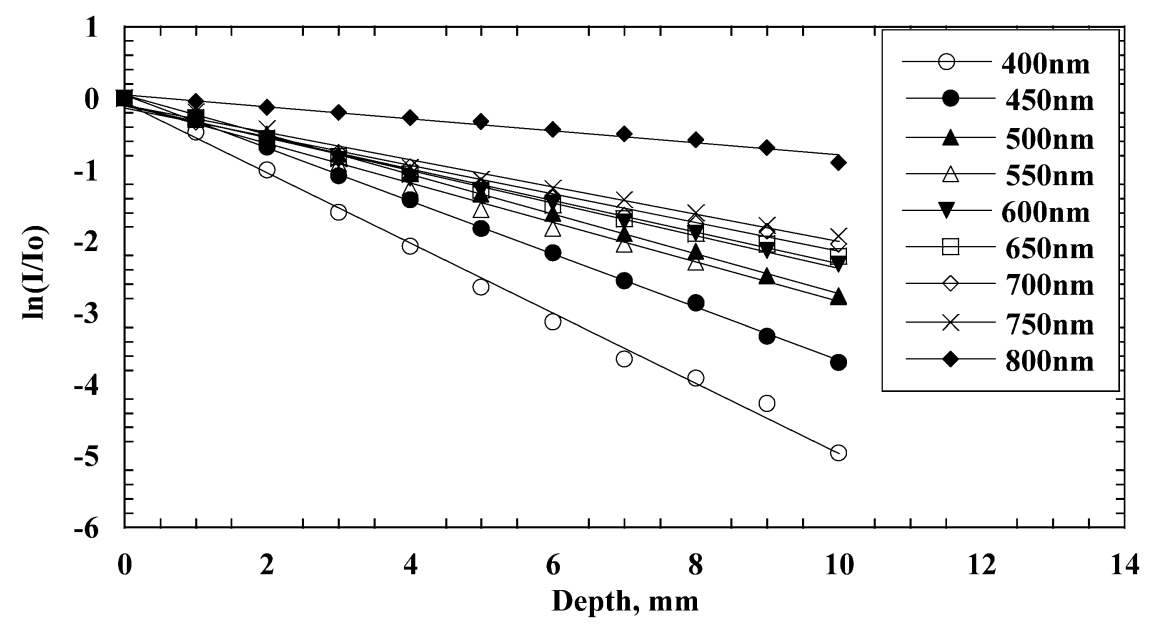

Fig. 5. Logarithm plot of visible light intensity versus depth in tissue phantom for different wavelengths.

exponential plot of Fig. 5 while the theoretical values are calculated by the specific relationship $\mathrm{HVL}_{\mathrm{th}}=$ $0.693 / \mu$. The calculations are based on assumption that the layers represent one bulk sample, so there is no variation in the refractive index in between. In contrast with Sprawls text book, the energy increases with increasing HVL while the linear attenuation coefficient is decreasing [4]. This contrast might be attributed to the radiation energy ranges. Low energies are usually absorbed in superficial tissues while the higher energy used by Sprawls is much higher and can penetrate the superficial region without attenuation. The values of $\mu_{\text {eff }}(0.22-0.42)$ in Table 1 are in very good agreement with the measurement of in vivo optical properties in animal models using implanted optical fibre detectors done by several researchers, Wilson et al. (0.26-0.48) [5], and Doiron et al. (0.16-0.23) in rabbit muscle [6], Wilson et al. (0.22-0.37) measured during PDT for human brain [7], and Wilson et al. (0.37-0.45) in pig brain [8], Drakaki et al. (0.01-0.4) in intralipid-agar with $12.4 \mu \mathrm{g} / \mathrm{ml}$ eosin at two visible wavelengths, 543 and $633 \mathrm{~nm}[9]$.

The optical penetration depth is the inverse function of the slope of the characteristic line $\left(\mu_{\mathrm{eff}}=1 / \delta\right)$. Since the slopes in Fig. 5 decrease with increasing wavelength where $(E \propto 1 / \lambda), \delta$ increases with increasing wavelength in the visible region of the light spectrum. Table 1 and Fig. 6 confirm this result. The optical penetration depth in the tissue increases with increasing wavelength. The high and low values around the $550 \mathrm{~nm}$ in Fig. 6 could be explained by the coffee mate absorption coefficient or due to the inhomogeneities of the tissue at this particular depth.

\section{2. $X$-ray}

The typical intensity-depth curves are shown in Fig. 7. The intensity of the X-ray radiation reveals a significant rise within the first few millimeters below the front surface of all tissue phantoms. The forward penetration becomes almost isotopic only in a region distal from the surface, and it is partially anisotropic near the surface.

Table 2, which shows the values of penetration depth $(\delta)$ using $20 \mathrm{keV}$ X-ray source, confirms these results. The average penetration depth in all the samples is $4.4-6 \mathrm{~mm}$, while the range of optical penetration depth is higher as compared to visible light $(0.8-3.3 \mathrm{~mm})$. One can conclude that the $\mathrm{X}$-ray radiation is not affected by the additives, while the penetration of visible light is totally dependent of additives. Furthermore, the final comparisons made between the two radiation sources are illustrated in Fig. 8. 


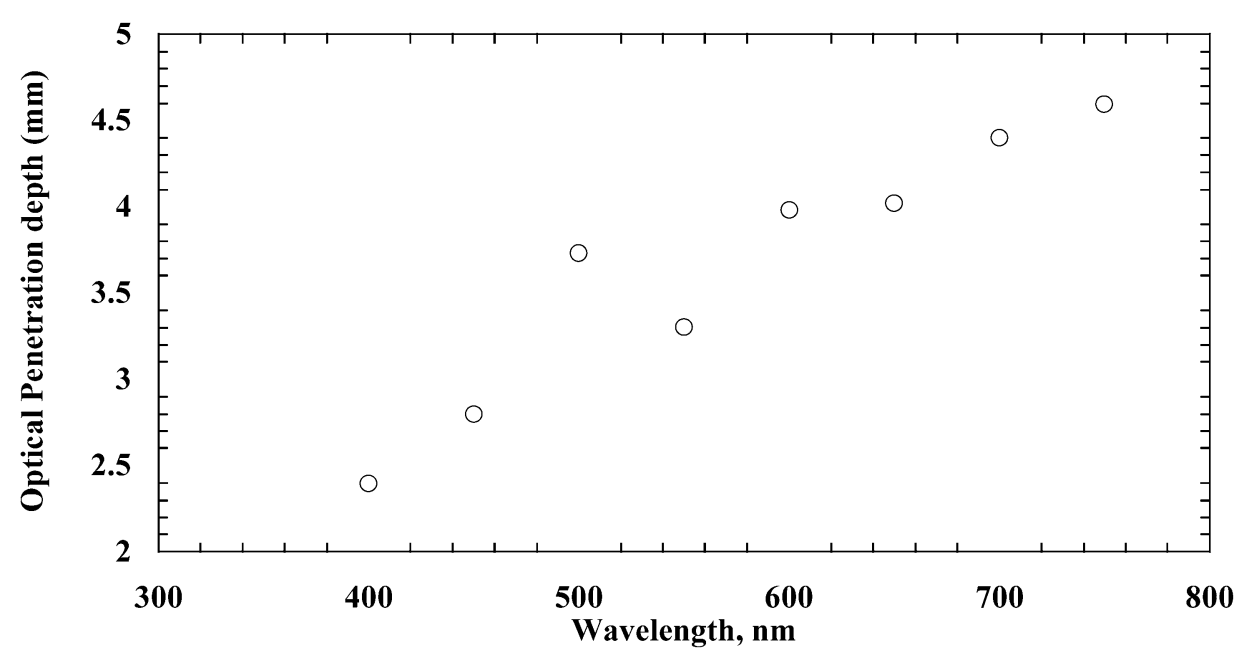

Fig. 6. The optical penetration depth in tissue phantom as a function of wavelength.

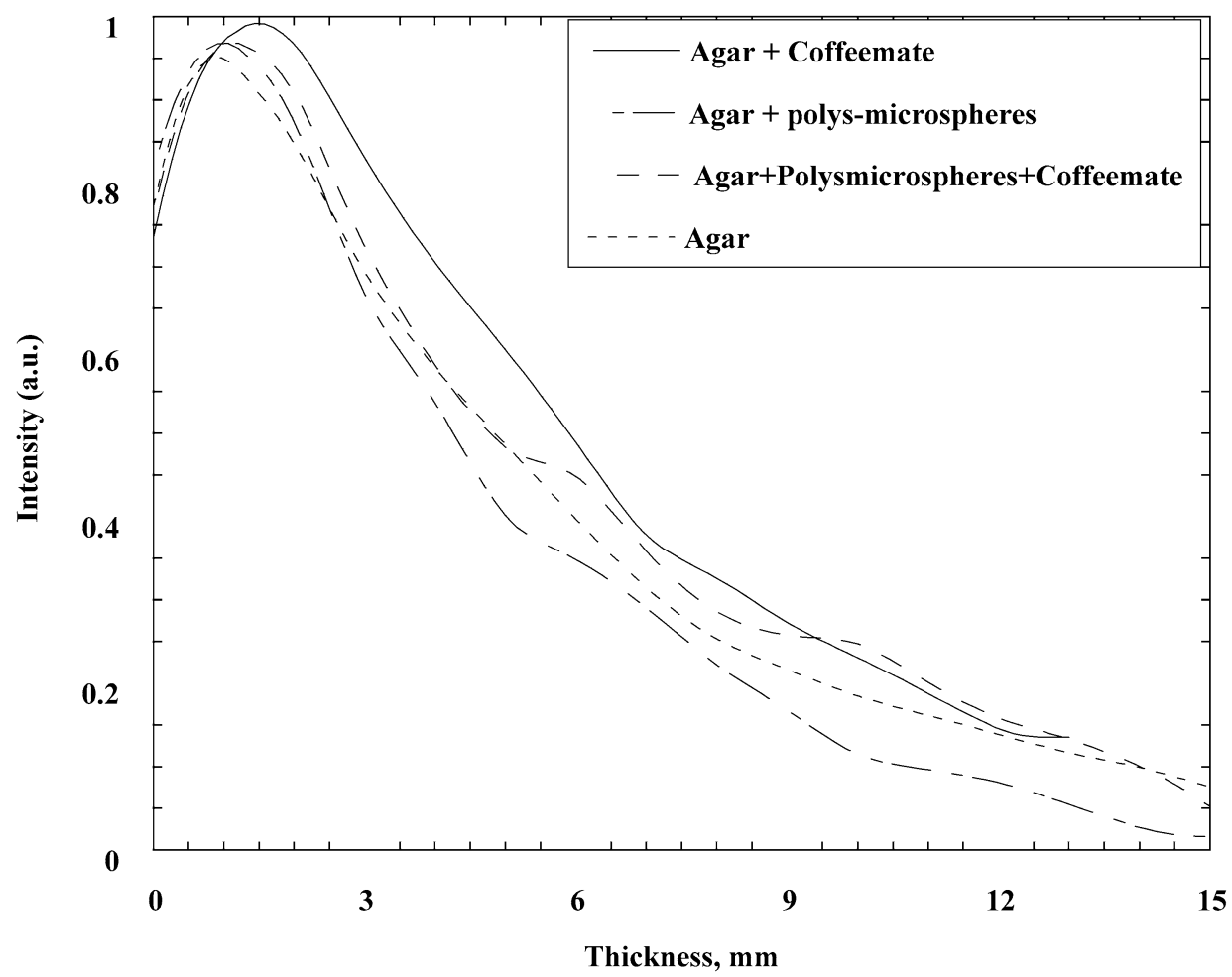

Fig. 7. Penetration of X-ray photon energy in tissue phantoms made of agar (as control), Agar + Coffee mate, Agar + polystyrene microspheres, and Agar + Coffee mate + polystyrene microspheres.

Figure 8 shows the higher penetration capability of X-ray, the photon beam fall off gradually with depth and never reach zero. In contrast, the visible light has a lower penetration in tissue. The penetration depths are X-ray $(6.19 \pm 0.009 \mathrm{~mm})$, and visible light $(3.32 \pm 0.005 \mathrm{~mm})$, where X-ray is higher than the visible light by 21 times. This information is consistent with the use of low energy in superficial therapy 
Table 2

Effect of additives on penetration depth $(\delta)$ in soft tissue phantom

\begin{tabular}{lcccc}
\hline Source & \multicolumn{3}{c}{$\delta(\mathrm{mm})$} \\
\cline { 2 - 5 } & $\begin{array}{c}\text { Agar } \\
(5.95 \mathrm{mg} / \mathrm{ml})\end{array}$ & $\begin{array}{c}\text { Agar }+ \text { Coffee } \\
\text { mate }(1.19 \mathrm{mg} / \mathrm{ml})\end{array}$ & $\begin{array}{c}\text { Agar + Polystyrene } \\
\text { microspheres }(0.3 \mu \mathrm{g} / \mathrm{ml})\end{array}$ & $\begin{array}{c}\text { Agar + Coffee mate } \\
+ \text { Polystyrene }\end{array}$ \\
\hline Visible light $(550 \mathrm{~nm})$ & $3.316 \pm 0.005$ & $1.437 \pm 0.044$ & $0.835 \pm 0.159$ & $0.918 \pm 0.040$ \\
X-ray $(20 \mathrm{keV})$ & $5.299 \pm 0.003$ & $5.995 \pm 0.009$ & $4.367 \pm 0.098$ & $5.724 \pm 0.008$ \\
\hline
\end{tabular}

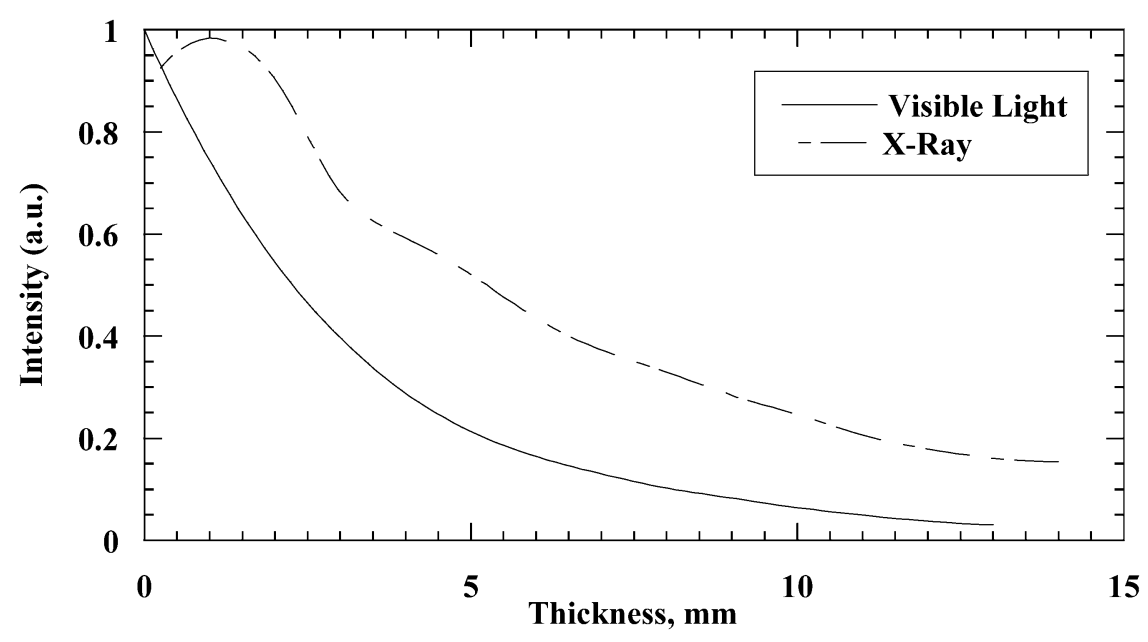

Fig. 8. Relationship between the X-ray, and light intensity versus layer depth in soft tissue.

Table 3

Influence of filtration on dose measured by film density using a diagnostic X-ray tube (40 keV)

\begin{tabular}{lcc}
\hline Filter & $\begin{array}{c}\text { Normalized density } \\
\text { (filter up) }^{\$}\end{array}$ & $\begin{array}{c}\text { Normalized density } \\
\text { (filter down) }^{\#}\end{array}$ \\
\hline No Filter & $1.000 \pm 0.015$ & $1.000 \pm 0.015$ \\
Aluminum only $(1 \mathrm{~mm})$ & $0.904 \pm 0.010$ & N.A. \\
Tissue only $(10 \mathrm{~mm})$ & $0.764 \pm 0.025$ & $0.804 \pm 0.006$ \\
Aluminum and tissue $(11 \mathrm{~mm})$ & $0.669 \pm 0.025$ & $0.728 \pm 0.005$ \\
\hline
\end{tabular}

* Results are expressed as means $\pm \mathrm{SD} .{ }^{\$}$ Filter is located at the exit window of the beam. ${ }^{\#}$ Filter is at the surface of the film $(\mathrm{SSD}=70 \mathrm{~cm})$.

and provides clear evidence and good support to such use, especially in photodynamic therapy.

The densities of the transmitted beam through the filters at the surface of film are shown in Table 3 . The densities were normalized to the non filter transmitted density. Significant reduction in the measured densities was noted when the filter is up (at the beam exit window). Whereas, $25 \%$ reduction were noticed when tissue used as a filter. Furthermore, 10 and 35\% reduction were noticed when Aluminum only or Aluminum plus tissue are used respectively. As tissue filter placed down (at the surface of the film) the densities are reduced by about $20 \%$. Density reductions of $28 \%$ were reported when tissue filter placed down and Aluminum filter placed up.

Figure $9(a-d)$ shows the effect of the filtration on the image quality, uniformity of distribution and structure identification of the phantom hand X-ray. Image quality, uniformity of distribution and structure 


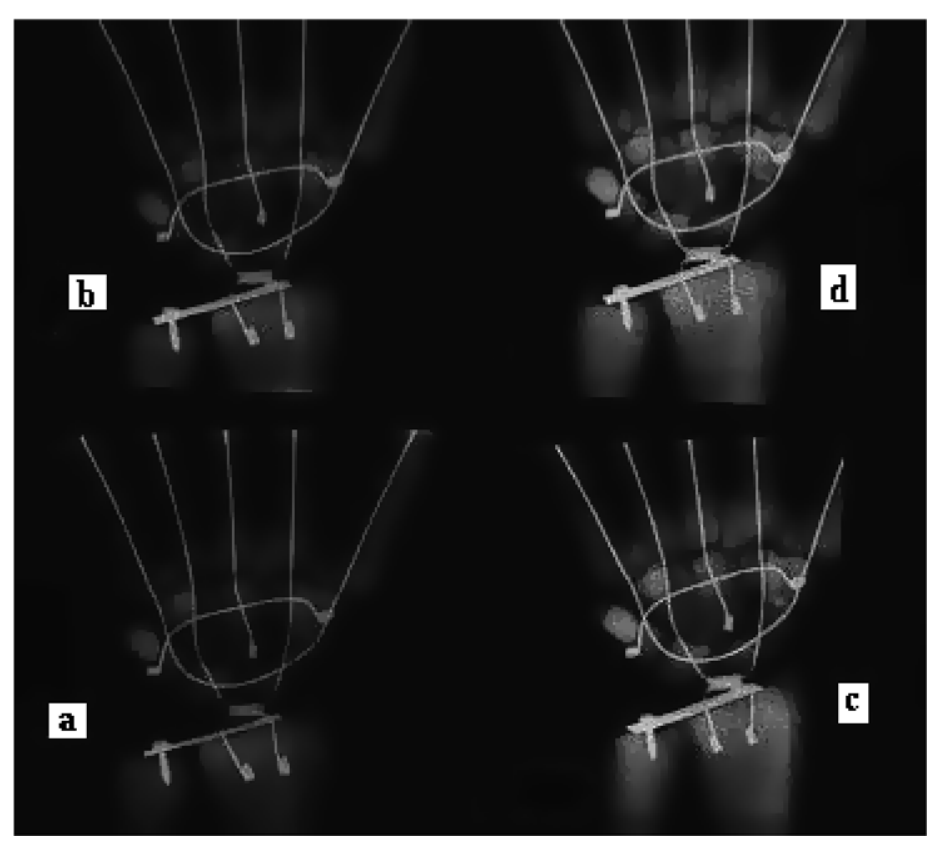

Fig. 9. X-ray film for hand-phantom with different filtrations: (a) no filtration, (b) $1 \mathrm{~mm}$ aluminium filter, (c) $10 \mathrm{~mm}$ tissue filter, and (d) $1 \mathrm{~mm}$ aluminium and $10 \mathrm{~mm}$ tissue filters.

identification using tissue filter alone (Fig. 9c) or a combination of both aluminum and tissue (Fig. 9d) are almost comparable and far much better than using no filter or using aluminum filter alone (Fig. 9(a and b)).

\section{Conclusions}

This research work can describes the production of an artificial cheap tissue phantom with well controlled tissue slicing of same thicknesses. The major advantage of this technique it can be used to simulate the biological tissue at different thicknesses using different wavelength and different energies. The results of this work might help to obtain a possible and accurate optical penetration depth of the human tissues in a wide range of energy spectrum, especially the extremely low energy. The values of $\mu_{\text {eff }}$ are in good agreement with those found by Wagnieres et al. This study emphasized the characteristics of the radiations interaction with tissues consist of some additives like absorber and scatterer materials to serve as a key and initial step for a wide range of radiation therapy diagnostic imaging.

Significant reduction in iterance dose and enhancement of the image could be achieved by using tissue filters. Further research work still is required to be done to measure some of useful parameters, like the scattering coefficient and absorption coefficient and compare it with well-known functions like Kubelka-Munk function [10-12]. Furthermore, precise mathematical modeling can be carried out to fit the biological changes to some useful formulas [13-15]. It would be interesting to investigate the dependence of the optical properties upon the concentration of materials in tissue phantom at specific wavelength. 


\section{Acknowledgements}

This work was financially supported by SURE program/United Arab Emirates University and Deanship of Scientific Research at Jordan University of Science and Technology (JUST). The investigators would also like to express their gratitude to the students Rouda Abdula Karim, Laila Al-Mazroeei, Dareen Mohamed, Karima Mohamed for their valuable time and great help. Great thanks to Dr. Fathallah Hamed for his useful comments and discussion.

\section{References}

[1] S. Bianco, F. Martelli and G. Zaccanti, Penetration depth of light re-emitted by a diffusive medium: theoretical and experimental investigation, Physics in Medicine and Biology 47 (2002), 4131-4144.

[2] J. Regan and J. Parrish, The Science of Photomedicine, Plenum Press, New York and London, 1982.

[3] G. Wagnières, S. Cheng, M. Zellweger, N. Utke, D. Braichotte, J. Ballini and V. Bergh, An optical phantom with tissuelike properties in the visible for use in PDT and fluorescence spectroscopy, Physics in Medicine and Biology 42 (1997), $1415-1426$.

[4] P. Sprawls, Physical Principles of Medical Imaging, 2nd edn, Medical Physics Publishing, Madison, WI, 1995.

[5] B.C. Wilson, W.P. Jeeves and D.M. Lowe, In vivo and post mortem measurements of the attenuation spectra of light in mammalian tissues, Photochemistry and Photobiology 42 (1985), 153-162.

[6] D.R. Doiron, L.O. Svaasand and A.E. Profio, Porphyrin Photosensitization, Plenum, New York, 1983, pp. 63-75.

[7] B.C. Wilson, P.J. Muller and J. Yanche, Instrumentation and light dosimetry for intra-operative photodynamic therapy (PDT) of malignant brain tumours, Medical Biology 31 (1986), 125-133.

[8] B.C. Wilson, M.S. Patterson, W.P. Jeeves, S.L. Flock and D.M. Lowe, Light propagation in tissue as applied to radiation dosimetry for photodynamic therapy, Medical and Biological Engineering and Computing 23(Suppl.) (1985), 213-256.

[9] E. Drakaki, S. Psycharakis, M. Makropoulou and A.A. Serafetinides, Optical properties and chromophore concentration measurements in tissue-like phantoms, Optics Communications 254 (2005), 40.

[10] L. Tremblay and J.P. Gagne, Fast quantification of humic substances and organic matter by direct analysis of sediments using DRIFT spectroscopy, Analytical Chemistry 74 (2002), 2985-2993.

[11] G. Kortüm, Reflectance Spectroscopy, Springer, Berlin, 1969.

[12] X. Zhang, L. Liang, J. Zhang and O. Su, Luminescence properties of (Ca1_xSrx)Se:Eu2+ phosphors for white LEDs application, Materials Letters $\mathbf{5 9}$ (2005), 749.

[13] M. Al-Akhras and L. Grossweiner, Sensitization of photohemolysis by hypericin and Photofrin ${ }^{\circledR}$, Journal of Photochemistry and Photobiology B: Biology 34 (1996), 169-175.

[14] M. Al-Akhras, A new application of Gompertz function in photohemolysis: The effect of temperature on red blood cell hemolysis photosensitized by protoporphyrin IX, Medical \& Biological Eng. \& Computing 44 (2006), 703-710.

[15] M. Al-Akhras, Effect of additives and photosensitive drugs on osmotic fragility, Int. J. Sci. Res. 16 (2006), 363-369. 


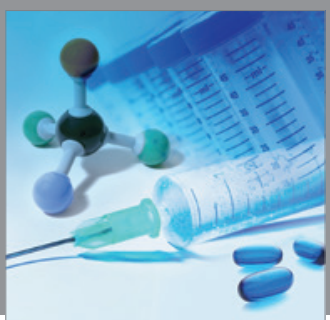

International Journal of

Medicinal Chemistry

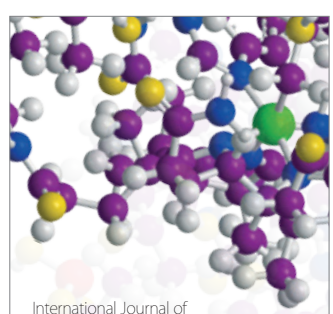

Carbohydrate Chemistry

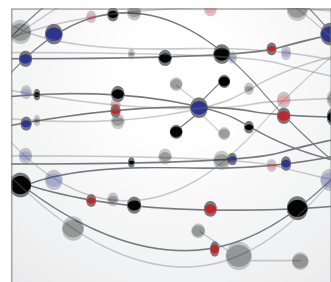

The Scientific World Journal
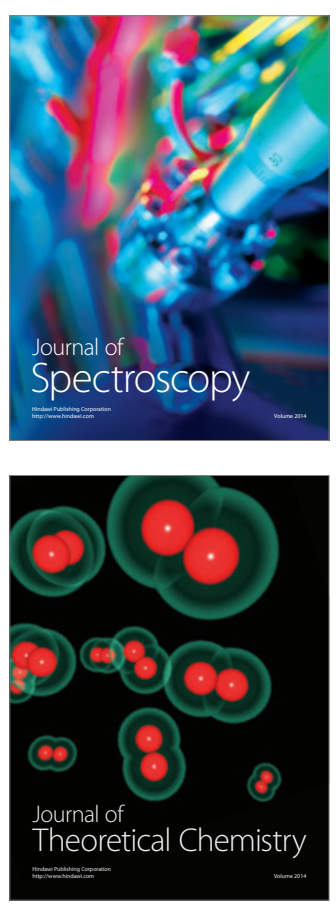
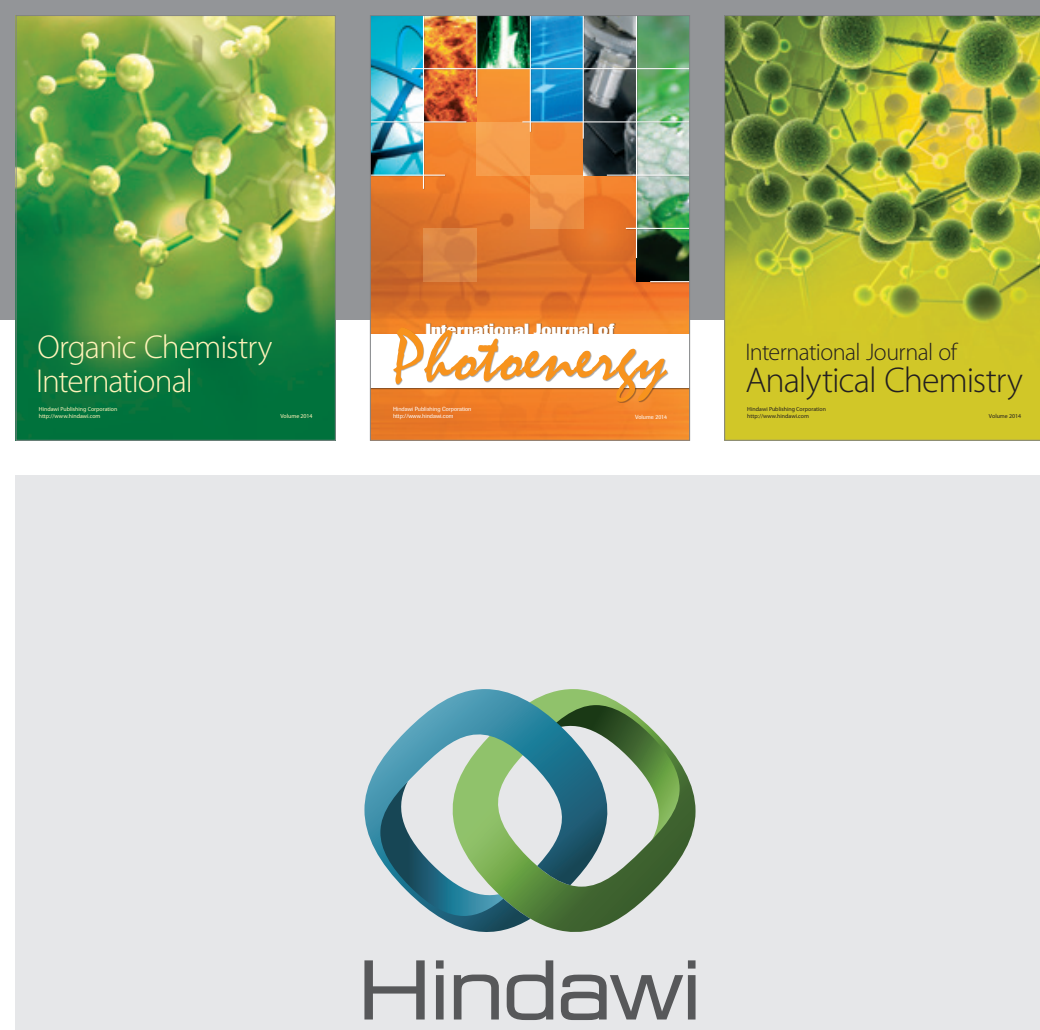

Submit your manuscripts at

http://www.hindawi.com
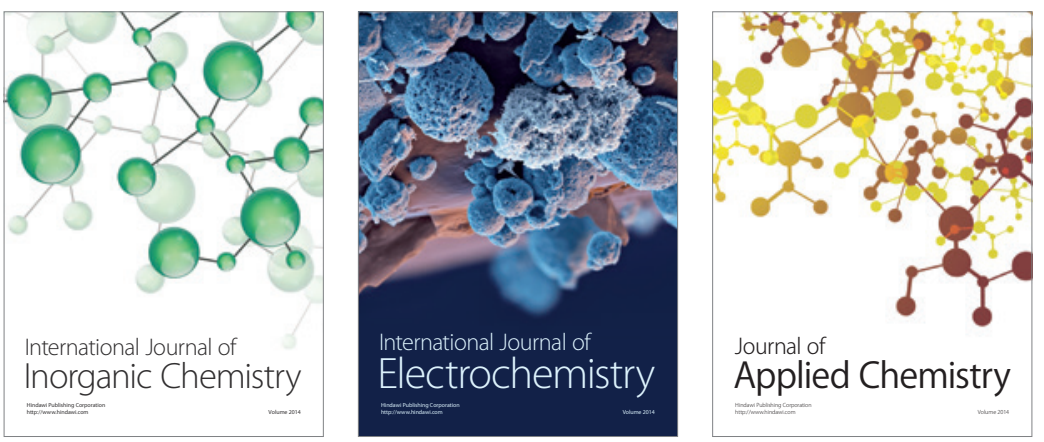

Journal of

Applied Chemistry
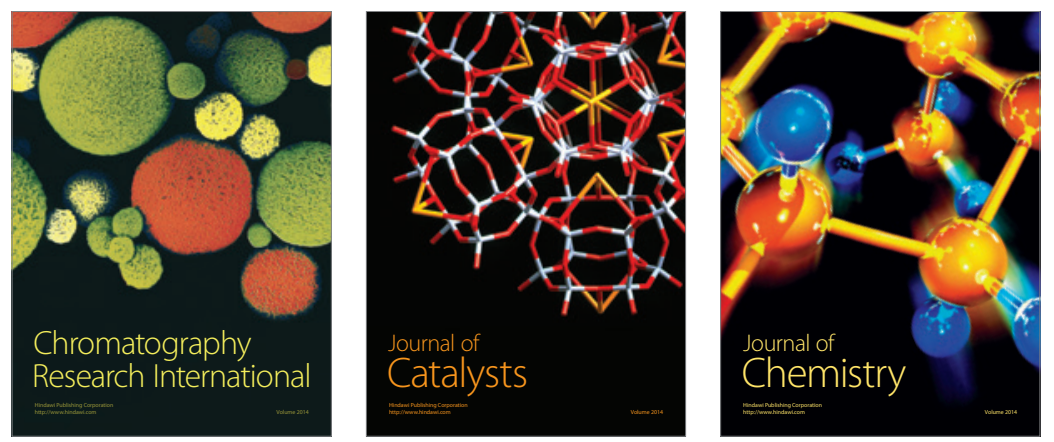
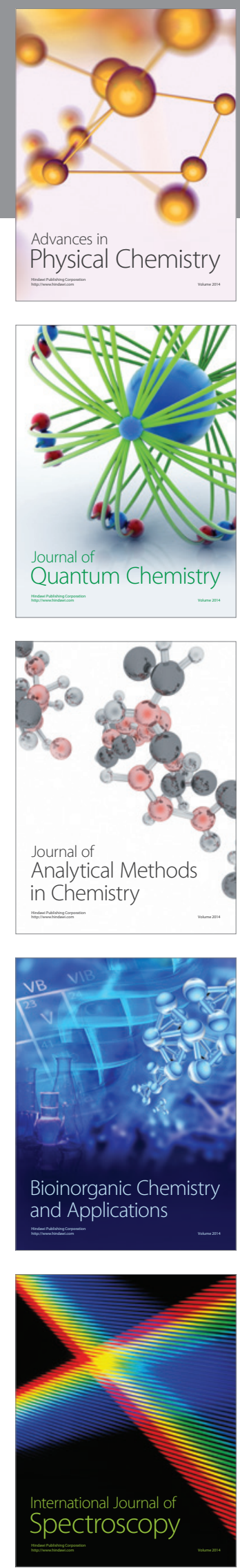